\title{
An innovative machine for Fused Deposition Modeling of metals and advanced ceramics
}

\author{
Hermes Giberti ${ }^{\mathrm{a}}$, Matteo Strano and Massimiliano Annoni \\ Dipartimento di Meccanica, Politecnico di Milano, Via La Masa 1, Milan, Italy
}

\begin{abstract}
The design of a new additive manufacturing (AM) system based on extrusion and 3D deposition of a mixture of metal (or advanced ceramic) powder and polymeric binder is described in this paper. The proposed system is totally innovative in terms of combination of deposited work material, extrusion system (head and nozzle), and deposition work table, which is based on a 5-axes parallel kinematics machine (PKM). The extrusion head and nozzle have been designed in order to be able to extrude high viscosity mixtures with low polymeric content. The 5-axes PKM is aimed at obtaining a good surface quality of the deposited work and reducing the need for supports during deposition. After the deposition, the material is de-binded and sintered to nearly the density of the solid material ascast. The design and kinematics of the machine and especially the PKM table is described in this paper, the main design issues are discussed and some preliminary extrusion and sintering results are presented.
\end{abstract}

\section{Introduction}

The design of a new additive manufacturing (AM) system based on extrusion and 3D deposition of a mixture of metal powder and polymeric binder will be described in this paper. The proposed system is innovative in terms of deposited work material, extrusion system, and kinematics. It is a technique for direct additive manufacturing of metal (or advanced ceramics) products. Direct-metal techniques can be classified as "Laminated manufacturing", "Powder bed processes" and "Deposition processes" [1].

FDM (Fused Deposition Modeling) is the most popular deposition process for plastic objects, but can hardly be used for advanced ceramics or metals. A recent review of extrusion based AM is given in reference [2]. Intense research is being developed worldwide in order to increase the applicability of material extrusion AM through the development of new materials [3]. Some authors have proposed the original FDM system for depositing a precursor filament of green ceramic [4] or a mixed metal/plastic filament [5]. When the percentage of ceramic component in the mixture is small, compared to the polymeric component, conventional FDM machines can be used [6]. However, some limitations are inherent to this process: the advantage of using a precursor filament is offset by problems encountered during its preparation and fabrication. During the extrusion, the frequent buckling failures cause interruption of the process; the backpressure encountered during the deposition limits the powder volume fraction in the filament, reducing the possibility of successful sintering of the produced part. Due to these problems, the FDM concept has been transformed by some authors, using as a starting material a ceramic clay or a metal slurry [7] instead of a plastic filament and replacing the torque and pinch system with a piston or screw injector [8] [9]. However, the use of a metal or ceramic slurry or feedstock still has some limitations, especially in terms of the minimum diameter of the extrusion nozzle. According to the Hagen-Poiseuille equation, a minor decrease in nozzle diameter dramatically decreases the flow rate and requires considerably greater pressures to extrude the slurry. For very small nozzle diameters (less than $0.3 \mathrm{~mm}$ ) and for viscous feedstock (with low binder percentage, less than $25 \%$ ), even complete blocking of the flow can be expected. The extrusion head can be powered up, but this would increase its weight and inertia and would make difficult to control the typical 2-axis motion of the extrusion head. For this reason, it seems that in case of FDM processes with heavy extrusion heads, a more convenient approach could be not moving the injection head ( $\mathrm{x}$ - and $\mathrm{y}$-axes) and the work table (z-axis), but moving only the table. This alternative approach has been very seldom explored in the scientific literature.

In this paper we propose an extrusion based system with 5-axes control of the work table and with parallel kinematics, which is unprecedented, at least to the authors' knowledge. The motivations for this innovative design are explained in this and in the following sections, but the main points are:

1) since the extrusion head is fixed in space, it poses no problem of positioning accuracy and it can be heavy and powerful;

2) since the kinematics is parallel, high positioning accuracy of the TCP (tool center point) can be expected;

\footnotetext{
${ }^{\mathrm{a}}$ Corresponding author: hermes.giberti@polimi.it
} 
3) since the tool-table orientation is controlled with 5 CNC axes, high surface quality can be expected and no or few supports are required while depositing.

The vast majority of industrial AM machines is designed and built on 3 degrees of freedom. Low cost, non-industrial additive manufacturing machines with extrusion heads, are all based on a 3-axes kinematic scheme. A typical limitation of traditional 3-axes AM processes is due to the scaled or irregular outer surface of the objects. It is an unavoidable stair-stepping effect related to the layered manufacturing principle [10]. In order to reduce this effect and to reduce the need for support structures the deposited part could be optimally oriented [11], [12]. In order to change the orientation of the part during the deposition, 5-axis and 6-axis AM machines are being increasingly used in deposition processes [13]. Some producers (e.g. Optomec, Efesto) propose laser based models which operate with 3-axes motions of the deposition head plus two rotational axes of the work-table.

At the same time, some examples of successful parallel kinematics approaches are being presented [14] that try to reduce the manufacturing costs. Parallel kinematics machines my also ensure precision, repeatability, stiffness and speed in positioning the table. Another advantage, when using kinematics with more than three axes, is that support structures, which are typical of extrusion-based additive systems, can be reduced or avoided for simple shapes.

\section{Description of the extrusion system and process}

Unlike in conventional FDM, the starting material is not in form of a wire, but in form of granules, fed by gravity through the feeding unit. The extrusion unit is shown in Fig. 1. A plasticizing screw, positioned at $45^{\circ}$ with respect to the vertical direction, continuously rotates and load with heated feedstock the injection cylinder. A vertical piston moves and pressurizes the heated feedstock through the injection nozzle. The diameter of the piston is $14 \mathrm{~mm}$. The injection pressure on the heated material, before the nozzle, can be set at a very high level, up to $140 \mathrm{MPa}$. The maximum amount of material that can be deposited during one single stroke of the piston is $9000 \mathrm{~mm} 3$; if an outlet wire of $0.6 \mathrm{~mm}$ is extruded, an approximate wire length of $32000 \mathrm{~mm}$ can be deposited before rechargeing the injection cylinder.

The deposition can be interrupted at any time by a computer controlled shutter. After shuttering, the piston retracts and in less than a second the screw fills again the cylinder with new material ready to be extruded. The proposed approach allows for high control of the extrusion speed and allows the injection of highly viscous material at an extrusion temperature of about $200^{\circ} \mathrm{C}$ with a minimum estimated wire diameter of $0.2 \mathrm{~mm}$.

Preliminary tests have been conducted with the extrusion unit. Two types of commercial feedstock materials have been used, both are intended for the MIM process. They are both made by a mixture of base powder materials (respectively stainless steel and zirconia) with water soluble thermoplastic binder. They both require, after extrusion, debinding and sintering operations. The elastic modulus of both materials, after injection molding and sintering is similar (about $200 \mathrm{GPa}$ ) but the ceramic is about four times harder than the stainless steel. The main initial, processing and final properties of both materials are given in Table 1.

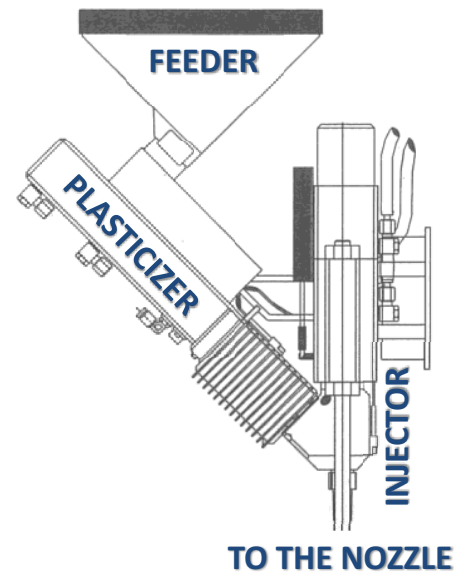

Figure 1. Proposed extrusion unit.

Table 1. Properties of materials used in extrusion tests.

\begin{tabular}{|l|l|l|}
\hline & Feedstock 1: metal & $\begin{array}{l}\text { Feedstock 2: } \\
\text { ceramic }\end{array}$ \\
\hline base material: & $\begin{array}{l}\text { martensitic } \\
\text { stainless steel AISI } \\
630, \text { nominal } \\
\text { density } 7.69 \mathrm{~g} / \mathrm{cm}^{3}\end{array}$ & $\begin{array}{l}\text { zirconia (ZrO2 at } \\
94.5 \% \text { ), partially } \\
\text { stabilized with } \\
\mathrm{Y}_{2} \mathrm{O}_{3}, \text { nominal } \\
\text { density } 6.04 \mathrm{~g} / \mathrm{cm}^{3}\end{array}$ \\
\hline binder & $\begin{array}{l}\text { water soluble } \\
\text { polyethylene } \\
\text { glycol, } \mathrm{b}_{\%}=21.0 \% \\
\text { in volume }\end{array}$ & $\begin{array}{l}\text { water soluble, } \\
\mathrm{b}_{\%}=15.8 \% \text { in } \\
\text { volume }\end{array}$ \\
\hline $\begin{array}{l}\text { debinding time } \\
\text { and maximum } \\
\text { temperature }\end{array}$ & $10 \mathrm{~h}, 60{ }^{\circ} \mathrm{C}$ & $\begin{array}{l}\text { about } 40 \text { hours, } \\
300{ }^{\circ} \mathrm{C}\end{array}$ \\
\hline $\begin{array}{l}\text { sintering time, } \\
\text { maximum } \\
\text { temperature, } \\
\text { atmosphere }\end{array}$ & $\begin{array}{l}1360{ }^{\circ} \mathrm{C}, \text { pure } \\
\text { hydrogen }\end{array}$ & $\begin{array}{l}\text { about } 29 \text { hours, } \\
1400{ }^{\circ} \mathrm{C}, \text { air }\end{array}$ \\
\hline $\begin{array}{l}\text { shrinkage after } \\
\text { sintering }\end{array}$ & $12.1 \%$ & $25.0 \%$ \\
\hline $\begin{array}{l}\text { hardness of } \\
\text { injection molded } \\
\text { and sintered } \\
\text { product }\end{array}$ & $>320 \mathrm{HV}$ & $>1200 \mathrm{HV}$ \\
\hline
\end{tabular}

\section{The robotic table}

Differently from what it is done in the most widespread scientific and industrial architectural solutions used in the field of additive manufacturing, in this conceptual design we propose to move the workpiece table instead of the extruder with five degrees of freedom. This layout allows us to obtain more flexibility in terms of the extrusion path generation.

Indeed in the AM applications the direction along which the material is extruded is supposed to be 
orthogonal to the plane on which it is deposited whereas by means of this solution it is possible to change the inclination of the workpiece-table and better follow the depositing task.

Our kinematic solution is based on a hybrid structure characterized by double parallel mechanisms (PKM). The first one has three translational degrees of freedom while the second is a two rotational DoFs manipulator. On the second platform is mounted the workpiece-table. The chosen hybrid kinematic solution allows one to reach good precision and a sufficiently wide working space. The platform has 5-DoF, three positioning DoF along the $\mathrm{x}, \mathrm{y}, \mathrm{z}$ axes, and two rotational DoF represented by the first two nautical angles, roll and pitch, considering an XYZ sequence. The five DoFs have been chosen in order to facilitate the deposition process of the feedstock and to allow A 3D deposition. The two manipulators are able to work in a coordinated way or independent of each other.

Figure 2 shows the scheme of the first parallel kinematics machine that constitutes the $3 \mathrm{~d}$ printer, named linear Delta. This architecture is equipped with three rigid links connected to the mobile platform to one side and to a linear guide on the other side.

The actuation is provided by three electrical motors joined to the three linear transmission units. Each link is made by a parallelogram scheme, able to reproduce a PUS (Prismatic-Universal-Spherical) kinematics chain. This solution ensures that the mobile platform is always parallel to the ground. In order to solve the kinematics of the machine, two different reference systems are defined: the first, inertial frame ( $x y z)$, is placed on the ground while the second, Tool-Center-Point (TCP) reference system, is linked to the mobile platform ( $\left.x^{\prime} y^{\prime} z^{\prime}\right)$. Note that due to the kinematic configuration, the axes of the two reference frames are always parallel in whatever pose of the robot.

By solving the inverse kinematics analysis, it is possible to evaluate each slider position $q_{i}$ along the guide from every TCP position. With reference to figure 2 for the $i$-th kinematic chain it's possible to state:

$\boldsymbol{l}_{i}=\boldsymbol{d}_{i}+q_{i} \widehat{\boldsymbol{u}}_{i}$ where $\boldsymbol{d}_{i}=\boldsymbol{p}+\boldsymbol{b}_{i}-\boldsymbol{s}_{i}$

by means of some simplifications it possible to state:

$q_{i}=\boldsymbol{d}_{i}^{T} \widehat{\boldsymbol{u}}_{i}-\sqrt{\boldsymbol{d}_{i}^{T}\left(\widehat{\boldsymbol{u}}_{i} \widehat{\boldsymbol{u}}_{i}^{T}-\boldsymbol{I}\right) \boldsymbol{d}_{i}+l_{i}^{2}}$

From the previous equation it is possible to find the relationship between the sliders and TCP velocities. It can be shown that the following expression holds:

$\widehat{\boldsymbol{n}}_{i}^{T} \widehat{\boldsymbol{u}}_{i} \dot{q}_{i}-\widehat{\boldsymbol{n}}_{i}^{T} \dot{\boldsymbol{p}}=0$

In the end, reorganizing the equations of the three kinematic chains in a matrix form, you get the following expression:

$\dot{\boldsymbol{q}}=\left[J_{q}\right]^{-1} \dot{\boldsymbol{p}}$

where $\left[J_{q}\right]^{-1}$ is the inverse of the Jacobian matrix of the translational motions.

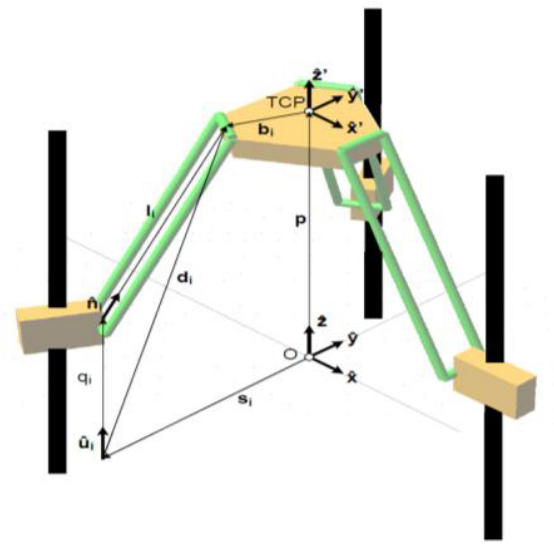

Figure 2. Linear delta scheme

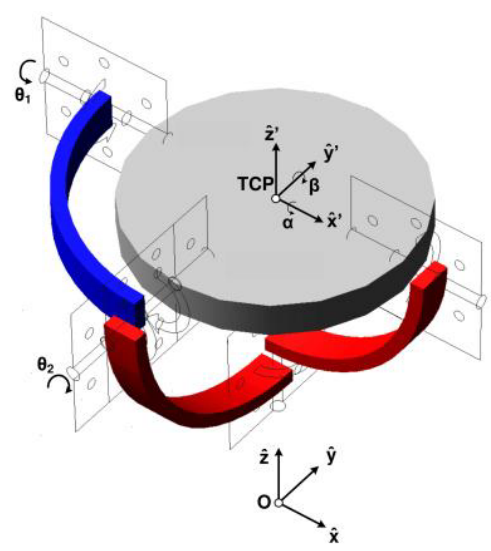

Figure 3. Two DoFs Agile Eye scheme

The second part of the 3D printer is based on a two rotational DoFs parallel kinematic manipulator named a spherical agile wrist. The scheme of this PKM, shown in Figure 3, is mounted on the linear Delta mobile platform. In order to solve the manipulator kinematics is necessary to evaluate the orientation of every one of its circular link. The expressions that describe the rotations of the motors $\left(\theta_{1}, \theta_{2}\right)$ in respect to the roll and pitch ( $\alpha, \beta$ respectively) of the platform are:

$$
\left\{\begin{array}{l}
\theta_{1}=\alpha \\
\theta_{2}=\tan ^{-1}(\cos \alpha \tan \beta)
\end{array}\right.
$$

Deriving these expressions with respect to time, you get:

$\dot{\boldsymbol{\Theta}}=\left[\begin{array}{cc}-\frac{s_{\alpha} t_{\beta}}{1+c_{\alpha}^{2} t_{\beta}^{2}} & \frac{c_{\alpha}\left(1+t_{\beta}^{2}\right)}{1+c_{\alpha}^{2} t_{\beta}^{2}} \\ 1 & 0\end{array}\right]\left\{\begin{array}{l}\dot{\alpha} \\ \dot{\beta}\end{array}\right\}=\left[J_{\theta}\right]^{-1}\left\{\begin{array}{l}\dot{\alpha} \\ \dot{\beta}\end{array}\right\}$

where $\dot{\boldsymbol{\Theta}}=\left\{\dot{\vartheta}_{1}, \dot{\vartheta}_{2}\right\}^{T}$ and the symbols $s_{x}, c_{x}, t_{x}$ represent respectively the sine, cosine and tangent of $x$, while $\left[J_{\theta}\right]^{-1}$ represents the inverse Jacobian matrix associated to the rotational motions.

\subsection{Driving System and Transmission Units}


In general parallel kinematic manipulators are not suitable for those applications in which a great workspace is required, while they represent the best choice when a high degree of precision is requiredAfter having optimised the working space of the robotic table, the mass of the bodies, which constitute the system, has been estimated and the driving system has been dimensioned. For this purpose, it has been necessary to solve the inverse dynamic, in order to evaluate the forces and the torques necessary to realize the desired TCP motion. By means of a multibody model of the robot it has been imposed a motion able to describe the worst operating conditions in which the system could be called to work. In these conditions, forces and torques, acting on the sliders, have been evaluated.

The model shown in Figure 4 has been developed using ADAMS. To probe the robot behaviour the projection of the workspace on the generic xy plan has been mapped by means of a grid of poses (each of them characterized by coordinates $\mathrm{x} 0 \mathrm{y} 0 \mathrm{z} 0$. Around each pose of the grid a sinusoidal motion has been imposed to the TCP in the three directions independently. The moments and force components perpendicular to the guide axis have been evaluated and their values have been compared with the maximum that this component is able to exert.

The sizing of the motoreducer group is based on the following equation:

$T_{m}=\tau T_{r}^{*}+J_{m} \frac{\dot{\omega}_{r}}{\tau}$ where $\mathrm{Tm}$ is the motor torque, $\mathrm{Jm}$ the motor inertia, $\tau$ the gear ratio provided by the reducer, and $\dot{\omega}_{r}$ the angular acceleration measured at the exit of the reducer. The term $T_{r}^{*}$ is an equivalent resistant torque which takes into account the inertia of the transmission unit, the friction between the slider and the guide and between the elements of the transmission unit, and the force component exerted by the robot along the guide axis. The results are computed in terms of maximum and RMS values in such a way that they can be compared with the motors data available on catalogues. So the equation (7) becomes:

$\alpha \geq \beta+\left[T_{r, r m s}^{*}\left(\frac{\tau}{\sqrt{J_{m}}}\right)-\dot{\omega}_{r, r m s}\left(\frac{\sqrt{J_{m}}}{\tau}\right)\right]^{2}$

where the quantities $\alpha$ and $\beta$ are introduced, following the theory described in [15-17] and particular $\left(\alpha=\frac{T_{m, n o m}^{2}}{J_{m}}\right)$ is the accelerating factor and depends on the motor characteristics only,

while $\left(\beta=2\left[\dot{\omega}_{r, r m s} T_{r, r m s}^{*}+\left(\dot{\omega}_{r} T_{r}^{*}\right)_{\text {mean }}\right]\right)$ is the load factor. From equation (8) it is possible to evaluate the range of the transmission ration $\tau$ for which the inequality is satisfied. Finally, the maximum torque and angular acceleration recorded during the motion must be lower compared to the maximum values achievable by the motor.

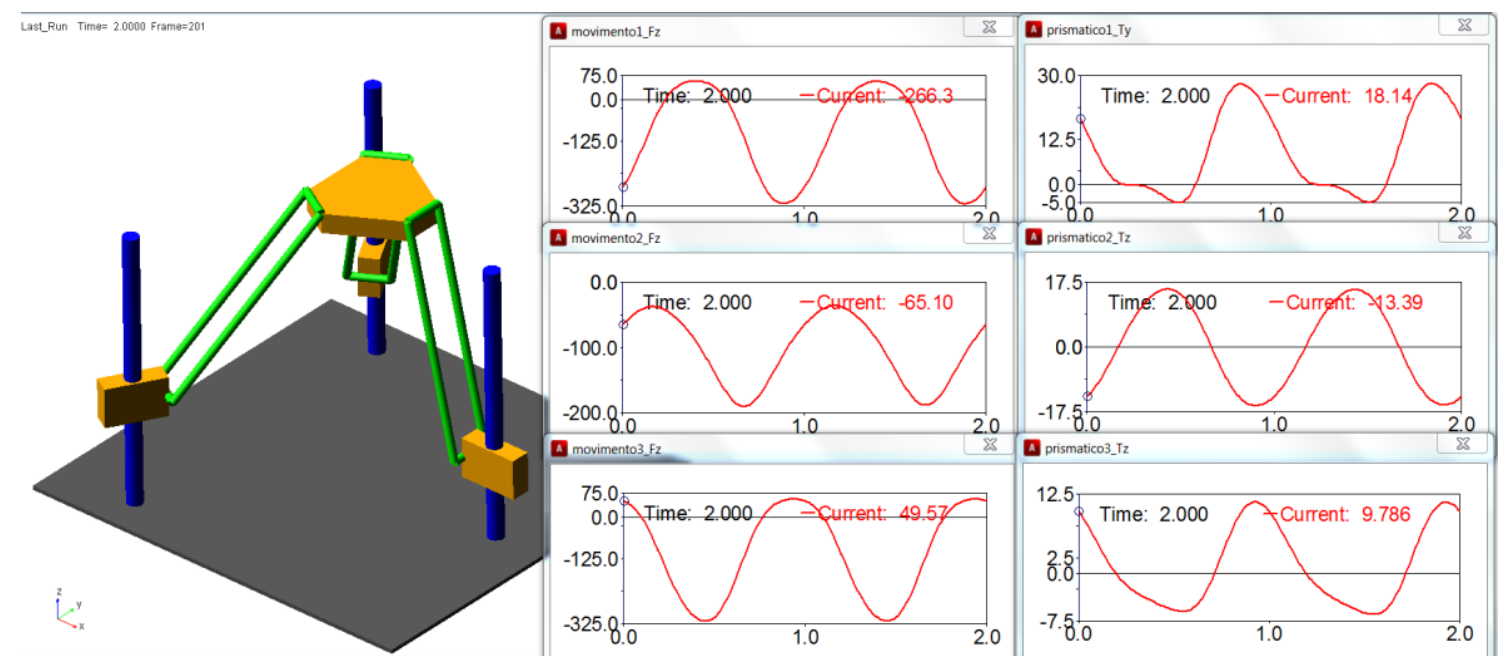

Figure 4. Multi-body model of the table 


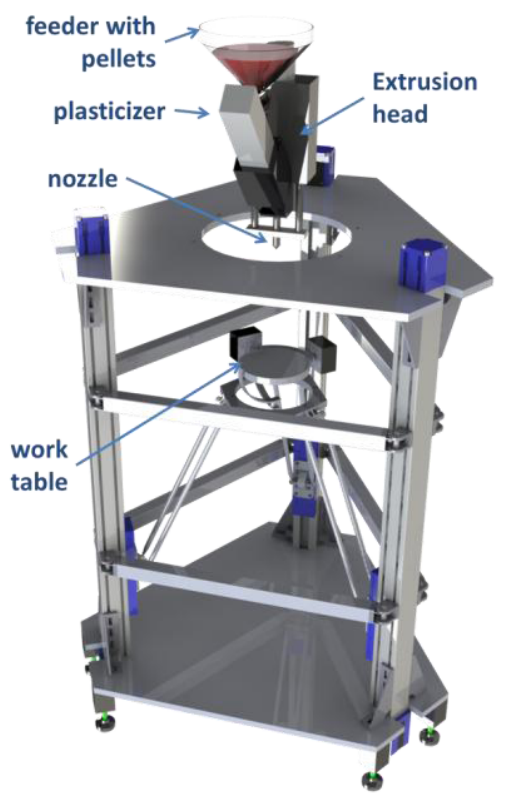

Figure 5. 3D printer conceptual design

\section{Machine integration}

By virtue of the above mentioned design tasks it has been possible to develop the conceptual design of the 3D printer shown in Figure 5. The system is able to move the TCP in a $250 \mathrm{~mm}$ cube with full orientation of the workpiece-table. The spherical wrist can rotate the end effector in a range of $+/-60^{\circ}$ degrees. The three linear guide $1500 \mathrm{~mm}$ length, have been used not only as the actuation system but also as the frame of the robot.

\section{Conclusions}

A revolutionary additive manufacturing (AM) system has been presented. If compared to conventional extrusionbased AM machines, the proposed system allows feeding through pellets rather than wire. A mixture of metal or ceramic powders with a very low percentage of thermoplastic binder makes the pellets [18]. The design of the extrusion head and nozzle has been discussed. The extrusion unit is fixed in space, due to its heavy weight, and the resulting extruded wire is deposited on a 5-axis moving table obtained with a parallel kinematics configuration. Currently, extrusion tests are being performed with different materials (stainless steel, zirconia, inconel), in order to find the optimal values of binder vs. powder load and in order to optimize the design of the nozzle. As a future step, the development of an application test case will be carried out, in order to evaluate the precision of the technology, the geometrical limits and the achievable surface roughness.

\section{References}

1. Karunakaran KP, Bernard A, Suryakumar S, Dembinski L, Taillandier G. Rapid manufacturing of metallic objects, Rapid Prototyp J (2012);18:264-80.
2. N. Turner B, Strong R, A. Gold S., A review of melt extrusion additive manufacturing processes: I. Process design and modeling, Rapid Prototyp J 2014;20:192-204.

3. Roberson D, Shemelya CM, MacDonald E, Wicker R., Expanding the applicability of FDM-type technologies through materials development, Rapid Prototyp J 2015;21:137-43.

4. Jafari MA, Mohammadi WH, Safari A, Danforth SC, Langrana N., A novel system for fused deposition of advanced multiple ceramics, Rapid Prototyp J 2000;6:161-75.

5. Masood S., Song W. Development of new metal/polymer materials for rapid tooling using fused deposition modelling, Mater Des 2004;25:58794.

6. Kalita SJ, Bose S, Hosick HL, Bandyopadhyay A. Developement of controlled porosity polymerceramic composite scaffolds via FDM Mater Sci Eng C 2003;23:611-20.

7. Li JP, de Wijn JR, Van Blitterswijk C a, de Groot K., Porous Ti6Al4V scaffold directly fabricating by rapid prototyping: preparation and in vitro experiment Biomaterials 2006;27:1223-35.

8. Bellini A, Shor L, Guceri SI., New developments in fused deposition modeling of ceramics, Rapid Prototyp J 2005;11:214-20.

9. Li J Bin, Xie ZG, Zhang XH, Zeng QG, Liu HJ. Key Eng Mater 2010;443:81-6.

10. Armillotta A., Assessment of surface quality on textured FDM prototypes, Rapid Prototyp J 2006;12:35-41.

11. Thrimurthulu K, Pandey PM, Venkata Reddy N., Optimum part deposition orientation in fused deposition modelling, Int $\mathrm{J}$ Mach Tools Manuf 2004;44:585-94.

12. Milewski J., Lewis G., Thoma D., Keel G., Nemec R., Reinert R., Directed light fabrication of a solid metal hemisphere using 5-axis powder deposition, J Mater Process Technol 1998;75:165-72.

13. Zhang J., Adaptive Slicing for a Multi-Axis Laser Aided Manufacturing Process, J Mech Des 2004;126:254.

14. Song X, Pan Y, Chen Y., Development of a LowCost Parallel Kinematic Machine for Multidirectional Additive Manufacturing, J Manuf Sci Eng 2014;137:021005.

15. Giberti H, Cinquemani S, Legnani G, Effects of transmission mechanical characteristics on the choice of a motor-reducer, Mechatronics 2010, 20, 604-610.

16. Giberti H, Cinquemani S, Legnani G, A practical approach to the selection of the motor-reducer unit in electric drive systems, Mechanics Based Design of Structures and Machines 2011, 39, 303-319.

17. Giberti H, Clerici A, Cinquemani S, Specific accelerating factor: One more tool in motor sizing projects, Mechatronics 2014, 24, 898-905.

18. Saggin B., Scaccabarozzi D., Tarabini M. Instrumental phase-based method for Fourier transform spectrometer measurements processing (2011) Applied Optics, 50 (12), pp. 1717-1725. 